\title{
Generalized Competing Glauber-type Dynamics and Kawasaki-type Dynamics
}

\author{
Han Zhu ${ }^{2}$, Jian-yang Zhu ${ }^{1,3^{*}}$ and Yang Zhou ${ }^{2}$ \\ ${ }^{1}$ CCAST (World Laboratory), Box 8730, Beijing 100080, China \\ ${ }^{2}$ Department of Physics, Nanjing University, Nanjing, 210093, China \\ ${ }^{3}$ Department of Physics, Beijing Normal University, Beijing 100875, China
}

(October 26, 2018)

\begin{abstract}
In this article, we have given a systematic formulation of the new generalized competing mechanism: the Glauber-type single-spin transition mechanism, with probability $p$, simulates the contact of the system with the heat bath, and the Kawasaki-type spin-pair redistribution mechanism, with probability $1-p$, simulates an external energy flux. These two mechanisms are natural generalizations of Glauber's single-spin flipping mechanism and Kawasaki's spin-pair exchange mechanism respectively. On the one hand, the new mechanism is in principle applicable to arbitrary systems, while on the other hand, our formulation is able to contain a mechanism that just directly combines single-spin flipping and spin-pair exchange in their original form. Compared with the conventional mechanism, the new mechanism does not assume the simplified version and leads to greater influence of temperature. The fact, order for lower temperature and disorder for higher temperature, will be universally true. In order to exemplify this difference, we applied the mechanism to $1 D$ Ising model and obtained analytical results. We also applied this mechanism to kinetic Gaussian model and found that, above the critical point there will be only paramagnetic phase, while below the critical point, the self-organization as a result of the energy flux will lead the system to an interesting heterophase, instead of the initially guessed antiferromagnetic phase. We studied this process in details.
\end{abstract}

PACS number(s): 05.50.+q, 05.70.Ln, 64.60.Cn, 64.60.Ht

\section{INTRODUCTION}

In recent years, there have been continuing efforts towards a clear picture of the self-organization phenomena in the phase transitions of magnetic systems. Most of the works [1 [ [] have been concentrated on Ising and Ising-like systems, governed by two competing dynamics: Glauber's single-spin flipping mechanism [9] and Kawasaki's spin-pair exchange mechanism [10], both with a probability. The system is coupled to a heat bath at a given temperature while being subject to an external energy flux. Glauber's flipping mechanism is to simulate the contact of the system with the heat bath. Changing the order parameter, it favors lower system energy. On the other hand, Kawasaki's exchange mechanism is to simulate the influence of the energy flux. Keeping the order parameter conserved, it favors higher system energy. With these two competing mechanisms and the corresponding master equation, people expect to obtain the evolution of the system. As exact treatment is not possible for $2 D$ Ising model, consequently Monte Carlo simulation and methods as Dynamic Pair Approximation have been employed. The results obtained helped to determine the interesting phase diagrams. (However, people are surprised to find there contradictions in the predictions of MC simulations and the dynamical mean-field theory, since they are both proved successful to yield good qualitative results in other studies. Though revisions of MC simulations are made and more accurate versions of mean-field theory are presented, the puzzle still remains [6].)

In our earlier studies, we have presented single-spin transition mechanism [11, 12] and spin-pair redistribution mechanism [13. These two dynamics are natural generalizations of Glauber's single-spin flipping mechanism and Kawasaki's spin-pair exchange mechanism respectively. They have similar mathematical expressions, and become counterparts of each other in the non-conserved and conserved processes respectively. As an example of the applications, we studied kinetic Gaussian model with both of them respectively. Our study shows that, in translational-invariant lattices, the dynamic critical exponent $z=1 / v=2$ is independent of space dimensionality and the governing dynamical mechanism. Its dynamic properties are summarized in Sec. IV.

In this article, we formulate the competing dynamics combining single-spin transition and spin-pair redistribution. As these two mechanisms themselves are universal, the combined one is also applicable to arbitrary systems, and it

\footnotetext{
*Author to whom correspondence should be addressed. Address correspondence to Department of Physics, Beijing Normal University, Beijing 100875, China. Email address: zhujy@bnu.edu.cn
} 
can be deemed as a generalization. In Sec. II, we first briefly review the two mechanisms respectively and then give the formulation of the competing mechanism. In Sec. III, we explain the differences between our mechanism and that adopted conventionally, taking $1 D$ Ising model as an example. In Sec. IV, as the chief task of this article, we apply it to kinetic Gaussian model and report the findings. In Sec. $\mathrm{V}$ we summarize our study with some discussions.

\section{THE COMPETING MECHANISM}

First we briefly review the single-spin transition mechanism and spin-pair redistribution mechanism.

\section{A. Single-spin transition mechanism}

Glauber's single-spin flipping mechanism allows an Ising system to evolve with its spins flipping to their opposite. In single-spin transition mechanism [11]12, a single spin $\sigma_{i}$ may change itself to any possible values, $\hat{\sigma}_{i}$, and the master equation is

$$
\frac{d}{d t} P(\{\sigma\}, t)=-\sum_{i} \sum_{\hat{\sigma}_{i}}\left[W_{i}\left(\sigma_{i} \rightarrow \hat{\sigma}_{i}\right) P(\{\sigma\}, t)-W_{i}\left(\hat{\sigma}_{i} \rightarrow \sigma_{i}\right) P\left(\left\{\sigma_{j \neq i}\right\}, \hat{\sigma}_{i}, t\right)\right],
$$

The transition probability is in a normalized form determined by a heat Boltzmann factor,

$$
W_{i}\left(\sigma_{i} \rightarrow \hat{\sigma}_{i}\right)=\frac{1}{Q_{i}} \exp \left[-\beta \mathcal{H}_{i}\left(\hat{\sigma}_{i}, \sum_{<i j>} \sigma_{j}\right)\right], Q_{i}=\sum_{\hat{\sigma}_{i}} \exp \left[-\beta \mathcal{H}_{i}\left(\hat{\sigma}_{i}, \sum_{<i j>} \sigma_{j}\right)\right] .
$$

One can clearly see that this mechanism favors a lower Hamiltonian of the system. Based on the master equation Eqs.(11), one can prove that the time expectations of single-spin and correlation functions are

$$
\begin{aligned}
& \frac{d}{d t}\left\langle\sigma_{i_{1}}(t) \sigma_{i 2}(t) \cdots \sigma_{i_{n}}(t)\right\rangle \\
= & -n\left\langle\sigma_{i_{1}} \sigma_{i_{2}} \ldots \sigma_{i_{n}}\right\rangle+\sum_{\{\sigma\}}\left\{\sum_{k=1}^{n}\left[\left(\prod_{j(\neq k)=1}^{n} \sigma_{i_{j}}\right)\left(\sum_{\hat{\sigma}_{i_{k}}} \hat{\sigma}_{i_{k}} W_{i_{k}}\left(\sigma_{i_{k}} \rightarrow \hat{\sigma}_{i_{k}}\right)\right)\right]\right\} P(\{\sigma\}, t) .
\end{aligned}
$$

When $n=1$, it is

$$
\frac{d}{d t} q_{k}(t)=-q_{k}(t)+\sum_{\{\sigma\}}\left[\sum_{\hat{\sigma}_{k}} \hat{\sigma}_{k} W_{k}\left(\sigma_{k} \rightarrow \hat{\sigma}_{k}\right)\right] P(\{\sigma\} ; t) .
$$

\section{B. Spin-pair redistribution mechanism}

Kawasaki's spin-pair exchange mechanism allows an Ising system to evolve with its nearest neighbors exchanging their spin values. In spin-pair redistribution mechanism [13], two neighboring spins, $\sigma_{j} \sigma_{l}$, may change to any possible values, $\hat{\sigma}_{j} \hat{\sigma}_{l}$, as long as their sum are conserved, and the master equation is

$$
\begin{aligned}
\frac{d}{d t} P(\{\sigma\}, t)= & \sum_{\langle j l\rangle} \sum_{\hat{\sigma}_{j}, \hat{\sigma}_{l}}\left[-W_{j l}\left(\sigma_{j} \sigma_{l} \rightarrow \hat{\sigma}_{j} \hat{\sigma}_{l}\right) P(\{\sigma\} ; t)\right. \\
& \left.+W_{j l}\left(\hat{\sigma}_{j} \hat{\sigma}_{l} \rightarrow \sigma_{j} \sigma_{l}\right) P\left(\left\{\sigma_{i \neq j}, \sigma_{l \neq k}\right\}, \hat{\sigma}_{j}, \hat{\sigma}_{l} ; t\right)\right] .
\end{aligned}
$$

The redistribution probability is also in a normalized form determined by a heat Boltzmann factor,

$$
W_{j l}\left(\sigma_{j} \sigma_{l} \rightarrow \hat{\sigma}_{j} \hat{\sigma}_{l}\right)=\frac{1}{Q_{j l}} \delta_{\sigma_{j}+\sigma_{l}, \hat{\sigma}_{j}+\hat{\sigma}_{l}} \exp \left[-\beta \mathcal{H}_{j l}\left(\hat{\sigma}_{j}, \hat{\sigma}_{l},\left\{\sigma_{m}\right\}_{m \neq j, l}\right)\right],
$$


where the normalization factor $Q_{j l}$ is

$$
Q_{j l}=\sum_{\hat{\sigma}_{j}, \hat{\sigma}_{l}} \delta_{\sigma_{j}+\sigma_{l}, \hat{\sigma}_{j}+\hat{\sigma}_{l}} \exp \left[-\beta \mathcal{H}_{j l}\left(\hat{\sigma}_{j}, \hat{\sigma}_{l},\left\{\sigma_{m}\right\}_{m \neq j, l}\right)\right] . .
$$

(Here it clearly favors a lower system Hamiltonian, but in the combined mechanism we shall change the sign before $\beta \mathcal{H}_{j l}$ and make it turn to the opposite.) The time expectation of single-spin is

$$
\frac{d}{d t} q_{k}(t)=-2 d q_{k}(t)+\sum_{w} \sum_{\{\sigma\}}\left[\sum_{\hat{\sigma}_{k}, \hat{\sigma}_{k+w}} \hat{\sigma}_{k} W_{k, k+w}\left(\sigma_{k} \sigma_{k+w} \rightarrow \hat{\sigma}_{k} \hat{\sigma}_{k+w}\right)\right] P(\{\sigma\} ; t),
$$

where $d$ is the space dimensionality and $\sum_{w}$ means a summation taken over the nearest neighbors.

\section{The competing mechanism}

With the competing mechanisms, single-spin transition with probability $p$ and spin-pair redistribution with probability $1-p$, the master equation can be written as,

$$
\frac{d}{d t} P(\{\sigma\}, t)=p G_{m e}+(1-p) K_{m e},
$$

where the Glauber-type

$$
G_{m e}=\sum_{i} \sum_{\hat{\sigma}_{i}}\left[-W_{i}\left(\sigma_{i} \rightarrow \hat{\sigma}_{i}\right) P(\{\sigma\}, t)+W_{i}\left(\hat{\sigma}_{i} \rightarrow \sigma_{i}\right) P\left(\left\{\sigma_{j \neq i}\right\}, \hat{\sigma}_{i} ; t\right)\right],
$$

and the Kawasaki-type

$$
\begin{aligned}
K_{m e}= & \sum_{\langle j l\rangle} \sum_{\hat{\sigma}_{j}, \hat{\sigma}_{l}}\left[-W_{j l}\left(\sigma_{j} \sigma_{l} \rightarrow \hat{\sigma}_{j} \hat{\sigma}_{l}\right) P(\{\sigma\} ; t)\right. \\
& \left.+W_{j l}\left(\hat{\sigma}_{j} \hat{\sigma}_{l} \rightarrow \sigma_{j} \sigma_{l}\right) P\left(\left\{\sigma_{i \neq j}, \sigma_{l \neq k}\right\}, \hat{\sigma}_{j}, \hat{\sigma}_{l} ; t\right)\right] .
\end{aligned}
$$

The Glauber-type mechanism is used to simulate the contact of the system with the external heat bath, and the transition probability is of the form given by Eqs.(2). This mechanism favors lower energy of the system. The spinpair redistribution mechanism is used to simulate the energy flux. The redistribution probability given above favors a lower system Hamiltonian, but what we need here is to the contrary. We can turn this tendency to its opposite if we change the sign before $\beta \mathcal{H}_{j l}$ in the redistribution probability. It has the following form,

$$
\begin{gathered}
W_{j l}\left(\sigma_{j} \sigma_{l} \rightarrow \hat{\sigma}_{j} \hat{\sigma}_{l}\right)=\frac{1}{Q_{j l}} \delta_{\sigma_{j}+\sigma_{l}, \hat{\sigma}_{j}+\hat{\sigma}_{l}} \exp \left[\beta \mathcal{H}_{j l}\left(\hat{\sigma}_{j}, \hat{\sigma}_{l},\left\{\sigma_{m}\right\}_{m \neq j, l}\right)\right], \\
Q_{j l}=\sum_{\hat{\sigma}_{j}, \hat{\sigma}_{l}} \delta_{\sigma_{j}+\sigma_{l}, \hat{\sigma}_{j}+\hat{\sigma}_{l}} \exp \left[\beta \mathcal{H}_{j l}\left(\hat{\sigma}_{j}, \hat{\sigma}_{l},\left\{\sigma_{m}\right\}_{m \neq j, l}\right)\right] .
\end{gathered}
$$

This normalized form implies that the tendency is toward a higher system Hamiltonian. The word, competition, is in fact that between the two opposite directions either favored by one mechanism.

As given above, we have already obtained the time expectation of single-spin with either mechanism respectively. One can prove that with competing mechanisms it will be,

$$
\frac{d}{d t} q_{k}(t)=p Q_{k}^{G}+(1-p) Q_{k}^{K}
$$

where the Glauber-type,

$$
Q_{k}^{G}=-q_{k}(t)+\sum_{\{\sigma\}}\left[\sum_{\hat{\sigma}_{k}} \hat{\sigma}_{k} W_{k}\left(\sigma_{k} \rightarrow \hat{\sigma}_{k}\right)\right] P(\{\sigma\} ; t)
$$


and the Kawasaki-type,

$$
Q_{k}^{K}=-2 d q_{k}(t)+\sum_{w} \sum_{\{\sigma\}}\left[\sum_{\hat{\sigma}_{k}, \hat{\sigma}_{k+w}} \hat{\sigma}_{k} W_{k, k+w}\left(\sigma_{k} \sigma_{k+w} \rightarrow \hat{\sigma}_{k} \hat{\sigma}_{k+w}\right)\right] P(\{\sigma\} ; t) .
$$

There are equations available for correlation functions with competing mechanisms, but in later studies we find this single-spin equation is enough to yield satisfying results.

\section{ON ISING MODEL}

As mentioned above, these years great efforts have been contributed to Ising model with competing dynamics. Because exact analytical treatment is too hard, most of the studies have been either approximation or Monte Carlo simulation. There are some differences between the conventional method and ours. In the following paragraph we present our considerations.

The results one may expect directly depend on the expression of the transition (flipping, exchange, redistribution) probabilities. We think that there are two requirements: First, this probability should contain the Hamiltonian, and thus naturally favors either higher energy (Kawasaki-type) or lower energy (Glauber-type). Second, introducing temperature into it, we require that the transition be influenced by the heat noise. It is the first requirement that makes the two mechanisms compete and in all the studies it has been well adopted. However, due to the difficulties of actual practice, most of them used the simplified versions. In most of the studies, the temperature factor has not been introduced into the exchange probability, while in the flipping probability it has been only partly combined. Typically for ferromagnetic Ising model it has been set as: Glauber-type

$$
W_{i}=\min \left\{1, \exp \left(\triangle E_{i} / K T\right)\right\},
$$

Kawasaki-type

$$
W_{i j}=\left\{\begin{array}{l}
1, \Delta E_{i j}>0 \\
0, \triangle E_{i j} \leqslant 0
\end{array} .\right.
$$

In the new mechanism the transition and redistribution probabilities do not take the simplified versions. Besides some mathematical aspects such as normalization, their difference lies in the role of temperature. With the new mechanism there is greater influence of heat on the system. The fact, order for lower temperature and disorder for higher temperature, is not universally true in the phase diagrams obtained in earlier studies [i 7]. However, we believe that this expectation will be unshakable if the system is governed by the new mechanism. In order to further study it, we applied our method to $1 D$ Ising model. It is well known that, due to the heat noise, there is only paramagnetic phase in $1 D$ Ising model. The analytical results we obtained confirms this conclusion, however one increases the energy flux, for all temperatures. (The details are in Appendix A, but we suggest you read it later for your easier understanding of our method.) $1 D$ Ising model governed by the conventional mechanism has been studied in Ref. [3] with approximation method and MC simulation. Further application on $2 D$ Ising model is beyond the scope of this article. There is no better or worse, since the two mechanisms have different characteristics, but we think the comparison will be interesting and also feasible in practice.

\section{ON THE KINETIC GAUSSIAN MODEL}

In this section we apply the new mechanism to the $3 D$ kinetic Gaussian model and report our findings with the phase diagram. One and two-dimensional cases are quite similar. First we briefly review some basic properties of this model.

Gaussian model, proposed by T. H. Berlin and M. Kac, at first in order to make Ising model more tractable, is an continuous-spin model. It has the same Hamiltonian as the Ising model (three dimensional),

$$
-\beta \mathcal{H}=K \sum_{i, j, k=1}^{N} \sum_{w} \sigma_{i j k}\left(\sigma_{i+w, j k}+\sigma_{i j+w, k}+\sigma_{i j, k+w}\right),
$$

where $K=J / k_{B} T$ and $\sum_{w}$ means summation over nearest neighbors. Compared with the Ising model, it has two extensions: First, the spins $\sigma_{i j k}$ can take any real value between $(-\infty,+\infty)$. Second, to prevent the spins from tending 
to infinity, the probability of finding a given spin between $\sigma_{i j k}$ and $\sigma_{i j k}+d \sigma_{i j k}$ is assumed to be the Gaussian-type distribution

$$
f\left(\sigma_{i j k}\right) d \sigma_{i j k}=\sqrt{\frac{b}{2 \pi}} \exp \left(-\frac{b}{2} \sigma_{i j k}^{2}\right) d \sigma_{i j k}
$$

where $b$ is a distribution constant independent of temperature. Being an extension of Ising model, Gaussian model is quite different however. In the equilibrium case, on translational invariant lattices it is exactly solvable, and later as a starting point to study the unsolvable models it has also been investigated with mean field theory and the momentum-space renormalization-group method.

As an example of the applications of single-spin transition and spin-pair redistribution mechanism, we have studied kinetic Gaussian model with both of them respectively. We summarize its dynamic properties as follows 111 13]. The inherent dynamical competition of this model is that: the system tries to lower its Hamiltonian with the spins tending to infinity, while the Gaussian-type probability serves to restrict this tendency. Above the critical temperature, the prevailing heat noise permits only a disordered state, whereas below the critical point some kind of order will appear. Our study shows that, on translational-invariant lattices, the dynamic critical exponent $z=1 / v=2$ is independent of space dimensionality and the governing dynamical mechanism.

Now we turn to treat the $3 D$ kinetic Gaussian model with the competing dynamics. $1 D$ and $2 D$ systems can be treated in a similar way and they have qualitatively the same properties. In earlier studies we obtained the time expectation of single-spin. With the competing mechanism we can borrow these equations from Ref. [11] and [13].

(1) With Glauber dynamics:

$$
\frac{d}{d t} q_{i j k}(t) \equiv Q_{i j k}^{G}=-q_{i j k}+\frac{K}{b}\left(q_{i-1, j, k}+q_{i+1, j, k}+q_{i, j-1, k}+q_{i, j+1, k}+q_{i, j, k-1}+q_{i, j, k+1}\right) .
$$

(2) With Kawasaki dynamics: since we have changed the sign before system Hamiltonian in the redistribution probability,

$$
\begin{aligned}
-\beta \mathcal{H} & =K \sum_{i, j, k=1}^{N} \sum_{w} \sigma_{i j k}\left(\sigma_{i+w, j k}+\sigma_{i j+w, k}+\sigma_{i j, k+w}\right), \\
\Longrightarrow \beta \mathcal{H} & =(-K) \sum_{i, j, k=1}^{N} \sum_{w} \sigma_{i j k}\left(\sigma_{i+w, j k}+\sigma_{i j+w, k}+\sigma_{i j, k+w}\right),
\end{aligned}
$$

all the expressions will remain the same if we switch $K$ to $-K$. Here we will have to do the same,

$$
\begin{aligned}
\frac{d}{d t} q_{i j k}(t) \equiv & Q_{i j k}^{K}=\frac{1}{2[b+(-K)]} b\left\{\left[\left(q_{i+1, j, k}-q_{i j k}\right)-\left(q_{i j k}-q_{i-1, j, k}\right)\right]\right. \\
& \left.+\left[\left(q_{i, j+1, k}-q_{i j k}\right)-\left(q_{i j k}-q_{i, j-1, k}\right)\right]+\left[\left(q_{i, j, k+1}-q_{i j k}\right)-\left(q_{i j k}-q_{i, j, k-1}\right)\right]\right\} \\
& +\frac{(-K)}{2[b+(-K)]}\left[2\left(2 q_{i-1, j, k}-q_{i-1, j+1, k}-q_{i-1, j-1, k}\right)+\left(2 q_{i-1, j, k}-q_{i j k}-q_{i-2, j, k}\right)\right. \\
& +2\left(2 q_{i+1, j, k}-q_{i+1, j+1, k}-q_{i+1, j-1, k}\right)+\left(2 q_{i+1, j, k}-q_{i j k}-q_{i+2, j, k}\right) \\
& +2\left(2 q_{i, j-1, k}-q_{i, j-1, k+1}-q_{i, j-1, k-1}\right)+\left(2 q_{i, j-1, k}-q_{i j k}-q_{i, j-2, k}\right) \\
& +2\left(2 q_{i, j+1, k}-q_{i, j+1, k+1}-q_{i, j+1, k-1}\right)+\left(2 q_{i, j+1, k}-q_{i j k}-q_{i, j+2, k}\right) \\
& +2\left(2 q_{i, j, k-1}-q_{i-1, j, k-1}-q_{i+1, j, k-1}\right)+\left(2 q_{i, j, k-1}-q_{i j k}-q_{i, j, k-2}\right) \\
& \left.+2\left(2 q_{i, j, k+1}-q_{i+1, j, k+1}-q_{i-1, j, k+1}\right)+\left(2 q_{i, j, k+1}-q_{i j k}-q_{i, j, k+2}\right)\right] .
\end{aligned}
$$

This expression is in fact not as complex as it seems. Each term in a bracket is a second order derivative of $q$, and they will cancel each other if a summation is taken over all the spins. Thus $\sum_{i j k} Q_{i j k}^{K}=0$.

Then with the competing mechanisms,

$$
\frac{d}{d t} q_{i j k}(t)=p Q_{i j k}^{G}+(1-p) Q_{i j k}^{K}
$$

It is natural to first consider using the system Hamiltonian, 


$$
\mathcal{H}=-J \sum_{\langle i, j\rangle} \sigma_{i} \sigma_{j}
$$

to characterize its behavior. If we find the Hamiltonian is decreasing, then the system is believed to be evolving to Ferromagnetic phase; if the Hamiltonian is stable around zero, the system must be in Paramagnetic phase; if the Hamiltonian is increasing, then the system is evolving towards Antiferromagnetic phase. Unfortunately, in Gaussian model although we can conveniently obtain the exact result for $\left\langle\sigma_{i} \sigma_{j}\right\rangle$ and the average value, $\sum_{i j}\left\langle\sigma_{i} \sigma_{j}\right\rangle / N^{2}$, we find it difficult to obtain an analytical result of the system Hamiltonian. In order to differentiate between these phases, instead we study the following two aspects. First, magnetization,

$$
M(t)=\frac{1}{N} \sum_{k} q_{k}(t),
$$

and second,

$$
M^{\prime}(t)=\frac{1}{N} \sum_{i j k} q_{i j k}^{\prime}(t)=\frac{1}{N} \sum_{i j k}(-)^{i+j+k} q_{i j k}(t)
$$

For pure ferromagnetic phase, if without any extra conditions, we expect nonzero magnetization, that is to say, $M(t) \neq 0$. At the same time, if the system is divided into two penetrating subsets, in one of which the spins have $i+j+k$ being an odd number, and in the other one the spins have $i+j+k$ being even, then one will find these two subsets are almost identical, and that leads to $M^{\prime}(t)=0$. So $M(t) \neq 0$ and $M^{\prime}(t)=0$ is the characteristic of ferromagnetic phase. For pure antiferromagnetic phase, we expect such a situation that the system can be divided into two penetrating opposing sublattices in the way mentioned above, one of positive spin and one of negative. Except the direction of the spins, these two sublattices are identical. This leads to $M^{\prime}(t) \neq 0$ but $M(t)=0$. In disordered paramagnetic phase, we expect disorder of the whole system and both $M(t)$ and $M^{\prime}(t)$ to be zero. Thus, if we get the evolution of $M(t)$ and $M^{\prime}(t)$, we can decide in which phase the system is.

With Eqs. 19), (20) and (21) we can write the time derivative of $M(t)$ and $M^{\prime}(t)$ as,

$$
\begin{aligned}
\frac{d M(t)}{d t} & =\frac{1}{N} \sum_{k} \frac{d}{d t} q_{k}(t) \\
& =p\left(-1+\frac{6 K}{b}\right) M(t) .
\end{aligned}
$$

and

$$
\begin{aligned}
\frac{d M^{\prime}(t)}{d t} & =\frac{1}{N} \sum_{k} \frac{d}{d t} q_{k}^{\prime}(t) \\
& =\left[-p\left(1+\frac{6 K}{b}\right)+6(1-p) \frac{6 K-b}{b-K}\right] M^{\prime}(t)
\end{aligned}
$$

The details of Eqs.(23) are in Appendix B. Because we want $K / b$ to be positive, the inequality

$$
-p\left(1+\frac{6 K}{b}\right)+6(1-p) \frac{6 K-b}{b-K}>0,
$$

becomes

$$
\frac{1}{12 p}\left(-36+41 p+\sqrt{\left(1296-2808 p+1561 p^{2}\right)}\right)<\frac{K}{b}<1,
$$

for $0<p<1$.

Fig.1 shows the phase diagram of $3 D$ kinetic Gaussian model. It is divided into several regions by the following three curves,

$$
\begin{gathered}
\exp (-K / b)=e^{-1 / 6}, \\
\exp (-K / b)=1 / e
\end{gathered}
$$


and

$$
\exp (-K / b)=\exp \left[-\frac{1}{12 p}\left(-36+41 p+\sqrt{\left(1296-2808 p+1561 p^{2}\right)}\right)\right] .
$$

Phase Diagrams for $1 D$ and $2 D$ models are quite similar.

We know that the critical point of $3 D$ Gaussian model is $K_{c}=b / 2 d$, where $d$ is the space dimensionality. In the region above line (25), the temperature is higher than the critical value. Both $M(t)$ and $M^{\prime}(t)$ are approaching zero exponentially, and this is identified as paramagnetic phase. The overwhelming heat noise permits no observable magnetization, and the Hamiltonian is static in equilibrium. When the temperature is below $T_{c}$, the heat noise becomes secondary and some kind of order appears.

For the region below line (25) and above curve (27), as well as that below line (26), we have exponentially increasing $M(t)$ while $M^{\prime}(t)$ is approaching zero, and this corresponds to ferromagnetic phase. In this phase, Glauber-type mechanism prevails and the energy is decreasing. There is observable homogeneous magnetization, the direction of which depends on that of the initial magnetization.

In the region between curve (26) and line (27) we have both exponentially increasing $M(t)$ and $M^{\prime}(t)$, and it can not be simply identified as ferromagnetic or antiferromagnetic. We give it a name, heterophase. In this region, the Kawasaki mechanism and the energy flux control the system, and the energy has a tendency to increase. The spin values are interesting. Our analytical results show that $M(t)=M(0) e^{A t}$ and $M^{\prime}(t)=M^{\prime}(0) e^{B t}$. This means that, if the initial phase is ferromagnetic, $M^{\prime}(0)=0$, but $M(0) \neq 0$, later we will observe $|M(t)|$ increasing but $M^{\prime}(t)$ staying at zero. If initially the system is antiferromagnetic, $M(0)=0$, but $M^{\prime}(0) \neq 0$, later we will have $\left|M^{\prime}(t)\right|$ increasing but $M(t)$ staying at zero. If initially a disordered paramagnetic phase is given, $M(0)=0$, and also $M^{\prime}(0)=0$, we will get both zero $M(t)$ and $M^{\prime}(t)$. From this one may get confused,- what is the real picture? How is the energy sure to increase if the spin values are dependent on other conditions? Direct computer simulation reveals the key. For example, we apply the periodic boundary condition and initially set the system as,

$$
\begin{aligned}
& \ldots+1,+1,-1,+1,+1,-1,+1,+1,-1,+1, \\
& +1,-1,+1,+1,-1,+1,+1,-1,+1,+1, \ldots
\end{aligned}
$$

This leads to $M(0) \neq 0$, but $M^{\prime}(0)=0$. After 1 second, it becomes (we only give approximate values),

$$
\begin{aligned}
& \ldots 0.7,1.1,0.2,0.8,0.6,0.3,0.6,0.6,0.2,0.6 \\
& 0.6,0.2,0.6,0.6,0.3,0.6,0.8,0.2,1.1,0.7, \ldots
\end{aligned}
$$

After 10 seconds,

$$
\begin{aligned}
& \ldots 9,13,5,17,3,17,3,15,6,11 \\
& 11,6,15,3,17,3,17,5,13,9, \ldots
\end{aligned}
$$

After 24 seconds,

$$
\begin{aligned}
& \ldots-249,6941,-6735,12089,-10041,13228,-8902,9922,-3755,3435, \\
& 3435,-3755,9922,-8902,13228,-10041,12089,-6735,6941,-249, \ldots
\end{aligned}
$$

This shows the routine of self-organization the system chooses in this specific case, and it is a self-explanatory picture of how the energy manages to increase while $M^{\prime}(t)=0$.

However, there is one exception. If at the beginning the system is set in a pure homogeneous ferromagnetic phase, we will find that the system remains in this homogeneous state. How can one explain this? Actually the system does have a tendency to increase its energy in this region, but it needs a hint to know exactly how. Here we cite the words of Tome and Oliveira [1]: It is "the result, in the case considered here, of a far from equilibrium process, namely, the continuous flux of energy into the system. Thus an instability of the usual (equilibrium) solutions leads the system toward states with spatial self-organized structure." The self-organization routine is closely related with a basic phenomenon of symmetry loss. A system under conditions that lead to paramagnetic phase is like a ball placed on the ground, for which all directions are identical. A system set in such a heterophase is like a ball placed on the top of another sphere. The upper ball has a tendency to fall down, but the direction depends on some kind of disturbance. If there is no disturbance at all, it will stay still. It will even have its energy increasing if the lower ball is being lifted up. Surely in reality we have never seen a ball stay still on the top of another, because disturbance can not be avoided. So discussion here is only to help qualitatively explain this strange phase and the mechanism hidden behind. 


\section{RESULTS AND SOME DISCUSSION}

In this article, we have given a systematic formulation of the new competing mechanism. The Glauber-type single-spin transition mechanism with probability $p$ simulates the contact of the system with the heat bath and the Kawasaki-type spin-pair redistribution mechanism with probability $1-p$ simulates an external energy flux. These two mechanisms themselves are natural generalizations of Glauber's single-spin flipping mechanism and Kawasaki's spinpair exchange mechanism. Thus, on the one hand, this mechanism is in principle applicable to arbitrary systems, while on the other hand, our formulation is able to contain a mechanism that just directly combines single-spin flipping and spin-pair exchange in their original forms (not simplified). Compared with the conventional one, the new mechanism does not assume the simplified versions. Their difference lies in the different role the system temperature plays. (As we have emphasized before, there is no better or worse, since they are different.) We applied the new mechanism to $1 D$ Ising model and used the analytical results to exemplify this difference. With the new mechanism, there is greater influence of temperature and the fact, order for lower temperature and disorder for higher temperature, will be universally true.

In Sec. IV, we applied this mechanism to $3 D$ kinetic Gaussian model. $1 D$ and $2 D$ models can be treated following a similar way and have qualitatively the same properties. We used $M(t)$ and $M^{\prime}(t)$ (their definition given in that section) to characterize the system. In the phase diagram of $3 D$ model, we confirm the expectation, order for lower temperature and disorder for higher temperature. For temperature above $T_{c}$, the system evolves to paramagnetic phase. For temperature lower than $T_{c}$, we observe ferromagnetic phase and another heterophase (instead of the antiferromagnetic phase as guessed). This interesting heterophase is the result of the energy flux and self-organization. We have analyzed it in details in that section, and hope it will help make clear the self-organization process in phase transitions. With regard to Ising model, we also hope it will be a good reference when people are trying to solve the puzzling differences between the results yielded by approximation method and MC simulation.

\section{ACKNOWLEDGMENTS}

This work was supported by the National Natural Science Foundation of China under Grant No. 10075025.

\section{APPENDIX A: CALCULATIONAL DETAILS ON THE KINETIC ISING MODEL}

We apply the new mechanism to $1 D$ Ising model. We derive the time expectation of single-spin using Eqs.(12),

$$
\frac{d}{d t} q_{k}(t)=p Q_{k}^{G}+(1-p) Q_{k}^{K}
$$

In this combined form, the Glauber-type term is as Eqs.(13),

$$
Q_{k}^{G}=-q_{k}(t)+\sum_{\{\sigma\}}\left[\sum_{\hat{\sigma}_{k}} \hat{\sigma}_{k} W_{k}\left(\sigma_{k} \rightarrow \hat{\sigma}_{k}\right)\right] P(\{\sigma\} ; t)
$$

and the Kawasaki-type is as Eqs.114,

$$
\begin{aligned}
Q_{k}^{K}= & -2 q_{k}(t)+\sum_{\{\sigma\}}\left[\sum_{\hat{\sigma}_{k}, \hat{\sigma}_{k+1}} \hat{\sigma}_{k} W_{k, k+1}\left(\sigma_{k} \sigma_{k+1} \rightarrow \hat{\sigma}_{k} \hat{\sigma}_{k+1}\right)\right. \\
& \left.+\sum_{\hat{\sigma}_{k}, \hat{\sigma}_{k-1}} \hat{\sigma}_{k} W_{k, k-1}\left(\sigma_{k} \sigma_{k-1} \rightarrow \hat{\sigma}_{k} \hat{\sigma}_{k-1}\right)\right] P(\{\sigma\} ; t) .
\end{aligned}
$$

First we calculate A1.

$$
\begin{aligned}
& \sum_{\hat{\sigma}_{k}} \hat{\sigma}_{k} W_{k}\left(\sigma_{k} \rightarrow \hat{\sigma}_{k}\right) \\
= & \frac{\sigma_{k} \exp \left[K \sigma_{k}\left(\sigma_{k-1}+\sigma_{k+1}\right)\right]-\sigma_{k} \exp \left[-K \sigma_{k}\left(\sigma_{k-1}+\sigma_{k+1}\right)\right]}{\exp \left[K \sigma_{k}\left(\sigma_{k-1}+\sigma_{k+1}\right)\right]+\exp \left[-K \sigma_{k}\left(\sigma_{k-1}+\sigma_{k+1}\right)\right]}
\end{aligned}
$$




$$
\begin{aligned}
& =\left\{\begin{array}{c}
0, \sigma_{k-1}=-\sigma_{k+1} \\
\tanh 2 K, \sigma_{k-1}=\sigma_{k+1}=1 \\
-\tanh 2 K, \sigma_{k-1}=\sigma_{k+1}=-1
\end{array}\right. \\
& =\frac{1}{2}\left(\sigma_{k-1}+\sigma_{k+1}\right) \tanh 2 K .
\end{aligned}
$$

So

$$
\begin{aligned}
Q_{G}^{k} & =-q_{k}(t)+\sum_{\{\sigma\}}\left[\sum_{\hat{\sigma}_{k}} \hat{\sigma}_{k} W_{k}\left(\sigma_{k} \rightarrow \hat{\sigma}_{k}\right)\right] P(\{\sigma\} ; t) \\
& =-q_{k}(t)+\frac{1}{2}\left(q_{k-1}+q_{k+1}\right) \tanh 2 K .
\end{aligned}
$$

Second, we calculate (A2),

$$
\begin{aligned}
& \sum_{\hat{\sigma}_{k}, \hat{\sigma}_{k+1}} \hat{\sigma}_{k} W_{k, k+1}\left(\sigma_{k} \sigma_{k+1} \rightarrow \hat{\sigma}_{k} \hat{\sigma}_{k+1}\right) \\
= & \frac{\sigma_{k} \exp \left[-K\left(\sigma_{k-1} \sigma_{k}+\sigma_{k+1} \sigma_{k+2}\right)\right]+\sigma_{k+1} \exp \left[-K\left(\sigma_{k-1} \sigma_{k+1}+\sigma_{k} \sigma_{k+2}\right)\right]}{\exp \left[-K\left(\sigma_{k-1} \sigma_{k}+\sigma_{k+1} \sigma_{k+2}\right)\right]+\exp \left[-K\left(\sigma_{k-1} \sigma_{k+1}+\sigma_{k} \sigma_{k+2}\right)\right]} \\
= & \left\{\begin{array}{c}
\left(\sigma_{k}+\sigma_{k+1}\right) / 2, \sigma_{k}=\sigma_{k+1} \\
\tanh \left[-K\left(\sigma_{k-1}-\sigma_{k+2}\right)\right], \sigma_{k}=-\sigma_{k+1}
\end{array}\right. \\
= & \frac{\sigma_{k}+\sigma_{k+1}}{2}+\frac{\left(\sigma_{k}-\sigma_{k+1}\right)^{2}}{4} \\
& \times\left[\frac{\left(\sigma_{k-1}-1\right)\left(\sigma_{k+2}+1\right)}{4}+\frac{\left(\sigma_{k-1}+1\right)\left(\sigma_{k+2}-1\right)}{-4}\right] \tanh (-2 K) . \\
= & \frac{\sigma_{k}+\sigma_{k+1}}{2}+\frac{1}{4}\left(\sigma_{k-1}-\sigma_{k+2}-\sigma_{k-1} \sigma_{k} \sigma_{k+1}+\sigma_{k} \sigma_{k+1} \sigma_{k+2}\right) \tanh (-2 K)
\end{aligned}
$$

And similarly,

$$
\begin{aligned}
& \sum_{\hat{\sigma}_{k}, \hat{\sigma}_{k-1}} \hat{\sigma}_{k} W_{k, k-1}\left(\sigma_{k} \sigma_{k-1} \rightarrow \hat{\sigma}_{k} \hat{\sigma}_{k-1}\right) \\
= & \frac{\sigma_{k}+\sigma_{k-1}}{2}+\frac{1}{4}\left(\sigma_{k+1}-\sigma_{k-2}-\sigma_{k+1} \sigma_{k} \sigma_{k-1}+\sigma_{k} \sigma_{k-1} \sigma_{k-2}\right) \tanh (-2 K) .
\end{aligned}
$$

So

$$
\begin{aligned}
Q_{k}^{K}= & -2 q_{k}(t)+\sum_{\{\sigma\}}\left[\sum_{\hat{\sigma}_{k}, \hat{\sigma}_{k+1}} \hat{\sigma}_{k} W_{k, k+1}\left(\sigma_{k} \sigma_{k+1} \rightarrow \hat{\sigma}_{k} \hat{\sigma}_{k+1}\right)\right. \\
& \left.+\sum_{\hat{\sigma}_{k}, \hat{\sigma}_{k-1}} \hat{\sigma}_{k} W_{k, k-1}\left(\sigma_{k} \sigma_{k-1} \rightarrow \hat{\sigma}_{k} \hat{\sigma}_{k-1}\right)\right] P(\{\sigma\} ; t) \\
= & \frac{1}{2}\left(q_{k-1}+q_{k+1}-2 q_{k}\right)+\frac{1}{4} \tanh (-2 K)\left(q_{k+1}-q_{k-2}+q_{k-1}-q_{k+2}\right) \\
& +\frac{1}{4} \tanh (-2 K)\left(\left\langle\sigma_{k} \sigma_{k+1} \sigma_{k+2}\right\rangle-2\left\langle\sigma_{k+1} \sigma_{k} \sigma_{k-1}\right\rangle+\left\langle\sigma_{k} \sigma_{k-1} \sigma_{k-2}\right\rangle\right) .
\end{aligned}
$$

Thus, combining (A5) and (A6) one will get,

$$
\begin{aligned}
\frac{d}{d t} q_{k}(t)= & p\left[-q_{k}(t)+\frac{1}{2}\left(q_{k-1}+q_{k+1}\right) \tanh 2 K\right] \\
& +(1-p)\left[\frac{1}{2}\left(q_{k-1}+q_{k+1}-2 q_{k}\right)+\frac{1}{4} \tanh (-2 K)\left(q_{k+1}-q_{k-2}+q_{k-1}-q_{k+2}\right)\right. \\
& \left.+\frac{1}{4} \tanh (-2 K)\left(\left\langle\sigma_{k} \sigma_{k+1} \sigma_{k+2}\right\rangle-2\left\langle\sigma_{k+1} \sigma_{k} \sigma_{k-1}\right\rangle+\left\langle\sigma_{k} \sigma_{k-1} \sigma_{k-2}\right\rangle\right)\right] .
\end{aligned}
$$


In order to decide in which phase the system is, we have suggested two quantities in Sec. IV,

$$
M(t)=\sum_{k} q_{k}(t)
$$

and

$$
M^{\prime}(t)=\sum_{k}(-)^{k} q_{k}(t) \equiv \sum_{k} q_{k}^{\prime}(t)
$$

We use their evolving tendency to characterize the system behavior. Obviously, (1) in a homogeneous ferromagnetic phase, we expect $|M(t)| / N \rightarrow 1$ and $\left|M^{\prime}(t)\right| / N \rightarrow 0 ;(2)$ in an antiferromagnetic phase consisting of two penetrating and opposing sublattices, we will have $\left|M^{\prime}(t)\right| / N \rightarrow 1$ and $|M(t)| / N \rightarrow 0$; (3) in disordered paramagnetic phase, both $M^{\prime}(t) / N$ and $M(t) / N$ will approach zero. (A detailed analysis can be found in that section.) With Eqs.(A7) we can get,

$$
\frac{d}{d t} M(t) \equiv \sum_{k} \frac{d}{d t} q_{k}(t)=-p(1-\tanh 2 K) M(t)
$$

Thus

$$
M(t)=M(0) \exp [-p(1-\tanh 2 K) t] .
$$

At the same time,

$$
\begin{aligned}
& \frac{d}{d t}(-)^{k} q_{k}(t) \\
\equiv & \frac{d}{d t} q_{k}^{\prime}(t)=p\left[-q_{k}^{\prime}(t)+\frac{1}{2}\left(-q_{k-1}^{\prime}-q_{k+1}^{\prime}\right) \tanh 2 K\right] \\
& +(1-p)\left[\frac{1}{2}\left(-q_{k-1}^{\prime}-q_{k+1}^{\prime}-2 q_{k}^{\prime}\right)+\frac{1}{4} \tanh (-2 K)\left(-q_{k+1}^{\prime}-q_{k-2}^{\prime}-q_{k-1}^{\prime}-q_{k+2}^{\prime}\right)\right. \\
& \left.+\frac{1}{4} \tanh (-2 K)\left(-\left\langle\sigma_{k}^{\prime} \sigma_{k+1}^{\prime} \sigma_{k+2}^{\prime}\right\rangle+2\left\langle\sigma_{k+1}^{\prime} \sigma_{k}^{\prime} \sigma_{k-1}^{\prime}\right\rangle-\left\langle\sigma_{k}^{\prime} \sigma_{k-1}^{\prime} \sigma_{k-2}^{\prime}\right\rangle\right)\right],
\end{aligned}
$$

and

$$
\begin{aligned}
M^{\prime}(t) & \equiv \sum_{k} \frac{d}{d t} q_{k}^{\prime}(t)=[p(-1-\tanh 2 K)+(1-p)(-2+\tanh 2 K)] M^{\prime}(t) \\
& =[-2+p+(1-2 p) \tanh 2 K] M^{\prime}(t) .
\end{aligned}
$$

Thus

$$
M^{\prime}(t)=M^{\prime}(0) \exp \{[-2+p+(1-2 p) \tanh 2 K] t\} .
$$

In $1 D$ Ising model, as given in Eqs.(A8) and $(\mathrm{A} 9), M(t)$ and $M^{\prime}(t)$ are both approaching zero exponentially, and we can make the conclusion that, however one increases the energy flux, the system will stay in paramagnetic phase at arbitrary temperature.

\section{APPENDIX B: CALCULATIONAL DETAILS OF EQS.(23)}

Kawasaki-type:

$$
\begin{aligned}
\frac{d}{d t} q_{i j k}^{\prime}(t)= & \frac{1}{2(b-K)} b\left\{\left[\left(-q_{i, j, k+1}^{\prime}-q_{i j k}^{\prime}\right)-\left(q_{i j k}^{\prime}+q_{i, j, k-1}^{\prime}\right)\right]\right. \\
& \left.+\left[\left(-q_{i+1, j, k}^{\prime}-q_{i j k}^{\prime}\right)-\left(q_{i j k}^{\prime}+q_{i-1, j, k}^{\prime}\right)\right]+\left[\left(-q_{i, j+1, k}^{\prime}-q_{i j k}^{\prime}\right)-\left(q_{i j k}^{\prime}+q_{i, j-1, k}^{\prime}\right)\right]\right\} \\
& +\frac{-K}{2(b-K)}\left[2\left(-2 q_{i-1, j, k}^{\prime}-q_{i-1, j+1, k}^{\prime}-q_{i-1, j-1, k}^{\prime}\right)+\left(-2 q_{i-1, j, k}^{\prime}-q_{i j k}^{\prime}-q_{i-2, j, k}^{\prime}\right)\right.
\end{aligned}
$$




$$
\begin{aligned}
& +2\left(-2 q_{i+1, j, k}^{\prime}-q_{i+1, j+1, k}^{\prime}-q_{i+1, j-1, k}^{\prime}\right)+\left(-2 q_{i+1, j, k}^{\prime}-q_{i j k}^{\prime}-q_{i+2, j, k}^{\prime}\right) \\
& +2\left(-2 q_{i, j-1, k}^{\prime}-q_{i, j-1, k+1}^{\prime}-q_{i, j-1, k-1}^{\prime}\right)+\left(-2 q_{i, j-1, k}^{\prime}-q_{i j k}^{\prime}-q_{i, j-2, k}^{\prime}\right) \\
& +2\left(-2 q_{i, j+1, k}^{\prime}-q_{i, j+1, k+1}^{\prime}-q_{i, j+1, k-1}^{\prime}\right)+\left(-2 q_{i, j+1, k}^{\prime}-q_{i j k}^{\prime}-q_{i, j+2, k}^{\prime}\right) \\
& +2\left(-2 q_{i, j, k-1}^{\prime}-q_{i-1, j, k-1}^{\prime}-q_{i+1, j, k+1}^{\prime}\right)+\left(-2 q_{i, j, k-1}^{\prime}-q_{i j k}^{\prime}-q_{i, j, k-2}^{\prime}\right) \\
& \left.+2\left(-2 q_{i, j, k+1}^{\prime}-q_{i+1, j, k+1}^{\prime}-q_{i-1, j, k+1}^{\prime}\right)+\left(-2 q_{i, j, k+1}^{\prime}-q_{i j k}^{\prime}-q_{i, j, k+2}^{\prime}\right)\right] .
\end{aligned}
$$

Glauber-type:

$$
\frac{d}{d t} q_{i j k}^{\prime}(t)=-q_{i j k}^{\prime}+\frac{K}{b}\left(-q_{i-1, j, k}^{\prime}-q_{i+1, j, k}^{\prime}-q_{i, j+1, k}^{\prime}-q_{i, j-1, k}^{\prime}-q_{i, j, k+1}^{\prime}-q_{i, j, k+1}^{\prime}\right) .
$$

And then

$$
\begin{aligned}
\frac{d M^{\prime}(t)}{d t} & =\frac{1}{N} \sum_{k} \frac{d}{d t} q_{k}^{\prime}(t) \\
& =\left[-p\left(1+\frac{6 K}{b}\right)+(1-p) 6 \frac{6 K-b}{b-k}\right] M^{\prime}(t)
\end{aligned}
$$

[1] T. Tome and M. J. de Oliveira, Phys. Rev. A 40, 6643 (1989).

[2] B. C. S. Grandi and W. Figueiredo, Phys. Rev. E 53, 5484 (1996), 54, 4722 (1996) 56, 5240 (1996), Phys. Rev. B 50, 12595 (1994).

[3] B. C. S. Grandi and W. Figueiredo, Mod. Phys. Lett. B, 10, 945 (1996).

[4] Yu-qiang Ma, Ji-wen Liu and W. Figueiredo, Phys. Rev. E 57, 3625 (1998).

[5] Ma Yuqiang and Liu Jiwen, Phys. Lett. A, 238, 159 (1998).

[6] Attila Szolnoki, Phys. Rev. E 62, 7466 (2000).

[7] S. Bekhechi, A. Benyoussef, B. Ettaki, M. Loulidi, and A. El Kenz, Phys. Rev. E 64, 016134 (2001).

[8] Mauricio Godoy and Wagner Figueiredo, Phys. Rev. E 65, 026111 (2002).

[9] R. J. Glauber, J. Math. Phys. 4, 294 (1963).

[10] K. Kawasaki, Phys. Rev. 145, 224 (1966).

[11] Jian-Yang Zhu and Z. R. Yang, Phys. Rev. E 59, 1551 (1999).

[12] Jian-Yang Zhu and Z. R. Yang, Phys. Rev. E 61, 210 (2000), Phys. Rev. E 61, 6219 (2000).

[13] Han Zhu and Jian-Yang Zhu, Kawasaki-type Dynamics: Diffusion in the kinetic Gaussian model, cond-mat/0204156.

\section{Caption of figures}

Fig.1. The phase diagram of $3 D$ Gaussian model with competing dynamics, Glauber-type with probability $p$, and Kawasaki-type with probability $1-p$. The system exhibits paramagnetic phase (Para), Ferromagnetic phase (Ferro), and heterophase (Hetero). 


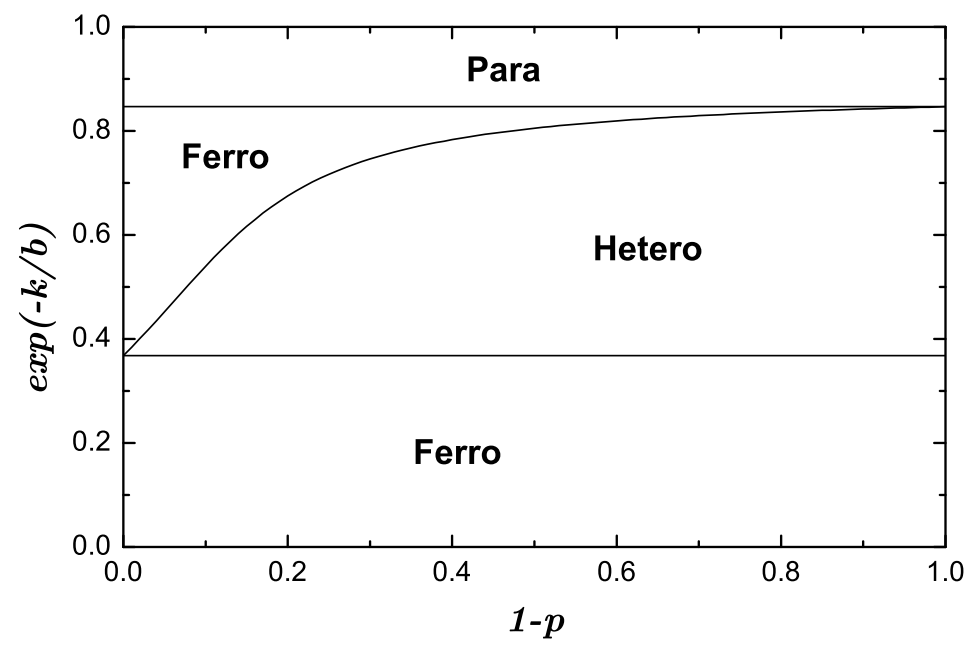

